\title{
Title: Advances in blood donor policies in relation to gay, bisexual and other men who have sex with men (gbMSM) and transgender populations as donors: $A$ scoping review.
}

Jacqueline Gahagan ( $\sim$ Jacqueline.Gahagan@msvu.ca )

Mount Saint Vincent University

Jennie Haw

Canadian Blood Services

Terrie Butler-Foster

Canadian Blood Services

Conor Falvey

Canadian Blood Services

Jackie Phinney

Dalhousie University

Marco Redden

Mount Saint Vincent University

Catherine Jenkins

Trans Care British Columbia

\section{Research Article}

Keywords: blood donation, plasma, gbMSM, transgender, sexual and gender minorities, health policy, health equity, Canada, OECD

Posted Date: February 28th, 2022

DOI: https://doi.org/10.21203/rs.3.rs-1217762/v1

License: (c) (1) This work is licensed under a Creative Commons Attribution 4.0 International License. Read Full License 


\section{Abstract}

Background: Globally, the updating of donor policies in relation to gay, bisexual and other men who have sex with men (gbMSM), transgender, and gender diverse populations remains an important public health issue. The purpose of this scoping review was to determine how Canada and other OECD countries are addressing this issue in relation to plasma and whole blood donation policies.

Methods: The scoping review included English-language peer-reviewed empirical studies from OECD countries from 1997 to 2020 to better understand the shifts in donor policies over this time period. Initial search criteria resulted in 3,974 abstracts and titles. Researchers reviewed abstracts according to eligibility criteria which yielded a total of 51 published studies for full review. Data charting was based on Arksey and O’Malley's framework for scoping reviews.

Results: Key considerations included the reliance on mathematical modeling and behavioural risk surveillance data as a means of continuing with the status quo in informing donor policies which continue to ban sexually active gbMSM from donating. In addition, there is a lack of focus on the unique concerns facing transgender, gender diverse, and racially diverse populations who may wish to donate whole blood or plasma.

Conclusions: There is a growing international policy shift in reducing the lifetime donor ban and/or removing the ban for gbMSM to donate blood. More needs to be done to both clarify the rationale for such policies in the face of current scientific evidence and testing and to ensure inclusion of transgender, gender diverse, and racially diverse populations in the formation of policies and information about both whole blood and plasma donations. Finally, blood operators need to work more closely with diverse donor communities to ensure the approach taken is in keeping with the needs of gbMSM, transgender, and gender diverse populations.

\section{Background}

\section{Context}

Blood operators around the world continue to address donor policies in relation to gay, bisexual and other men who have sex with men (gbMSM) and transgender populations that are viewed by many as discriminatory. Globally, these policies range from an outright ban on any form of blood donation by sexually active gbMSM, deferrals after sexual activity before gbMSM can donate, to individual behaviourbased screening approaches that enable some sexually active gbMSM to donate.

In Canada, a 3-month deferral period for sexually active gbMSM has been in place since 2019. In Spring 2021, Canadian Blood Services (CBS) made a submission to Health Canada to enable some sexually active gbMSM identified as "low risk" to donate apheresis plasma for fractionation in two collection centres. The proposed program would include additional sexual behaviour questions for gbMSM in the screening questionnaire to identify donors who may be eligible to donate. While many view this program 
as insufficient in addressing discriminatory blood donation policies, proponents argue that this offers an initial step towards enabling sexually active gbMSM to donate blood. CBS has announced its intention to make a further submission to Health Canada in Fall 2021 recommending an end to waiting periods for gbMSM donors for all blood products and screening all donors according to specific sexual behaviours (1).

There has been significant treatment of the equity issues at play in blood donation policies from social science and/or humanities perspectives. Aspects of equity that have been examined include sexual orientation (2,3), racialized identities/Blackness (4), and transgender identities $(5,6)$. However, there is a dearth of peer reviewed primary scientific literature regarding blood and plasma donation dealing with equity considerations in policy development. In particular, there is virtually no such research on transgender donors. However, negative and stigmatizing donation experiences are readily reported in the media (5). In addition, there is no worldwide consensus on donation eligibility criteria for transgender donors. For example, some blood operators report that transgender donors are ineligible entirely from donation while other blood operators report differential screening practices for transgender donors including registering donors in the sex assigned to them at birth, and asking donors about lower genital gender affirming surgery in order to determine which of the gendered "MSM questions" should be asked. Other blood operators do not report their screening for transgender donors openly (7). The male/female binary intake systems at blood centres, focus on gendered screening questions, and asking invasive questions only to transgender donors has been heavily criticized in grey literature and media (8-10) but is largely absent from academic donor policy discourse (5).

In Canada, transgender and non-binary donors are required to identify within a gender binary, as either " $\mathrm{M}$ " or " $\mathrm{F}$," and are registered according to the sex assigned to them at birth unless they have had lower genital gender affirming surgery. The Fall 2021 Health Canada submission will include a request to remove the gender-affirming surgery question and enable transgender donors to register according to their self-identified gender within the limits of a gender binary intake system. This change will not address the erasure of donors whose gender is not recognized within the binary. Current IT limitations prohibit the inclusion of multiple gender categories in computerized donor management systems.

The absence of transgender, non-binary, and gender diverse participants from the primary scientific literature that informs these policy discussions is cause for concern, as is the lack of attention to other dimensions of equity such as racial diversity. Further data on these populations is necessary to ensure informed, effective, and equity-conscious policymaking by blood operators, including Canadian Blood Services. This scoping review, the first phase of a larger study, aims to contribute to this work by reviewing the scientific literature on whole blood and plasma donation by gbMSM in other OECD countries.

\section{Objectives}


This scoping review examines the empirical research informing donor policies in OECD countries, assesses the type of research and perspective taken, and identifies key gaps in the literature. The aim is to use these results to inform recommendations for blood operators. This scoping review is intended to form the initial phase of a larger national research project that will assess the awareness of and attitudes towards the proposed screening and eligibility changes among Canadian gbMSM and transgender communities. The second phase will comprise qualitative data collection within gbMSM, transgender, non-binary, and gender diverse communities in Canada, while the third phase will include an online quantitative survey of these same communities.

This scoping review focuses on technologies, processes, and policies for gbMSM and transgender populations in relation to apheresis plasma and whole blood donation. To date, no scoping review has been conducted on these topics despite the various challenges and proposed changes to policies taking place in many OECD countries.

There is growing international support for removing the current ban on blood donations from gbMSM and implementing an individual behaviour-based screening approach for all donors. Moreover, there is indication that some blood operators are increasingly aware of the need to address policies and practices that do not meet the needs of transgender and gender-diverse donors (7). Given these issues, empirical research that may inform these donor policy revisions are all the more relevant. In this context, an assessment of the research that is available is both timely and necessary to inform donor policies going forward.

\section{Methods}

\section{Eligibility criteria}

The scoping review included only English-language articles that reported empirical studies and were published in peer-reviewed journals since 1997. To be included, studies had to pertain to an OECD country, focus on gbMSM or transgender blood and/or plasma donation, and include at least one relevant phenomenon and at least one relevant outcome. The relevant phenomena of interest were blood donation or apheresis plasma donation policies; blood donation screening questions; or technologies, policies, processes or programs to reduce blood-borne pathogens from whole blood donation. The relevant outcomes were acceptability of blood donation and apheresis plasma donation policies and programs; understanding and awareness of apheresis plasma donation; or blood or apheresis plasma donation eligibility expansion.

\section{Information sources and search strategy}


A medical librarian (JP) constructed a comprehensive search strategy that included both keywords and subject headings (exploded where appropriate) surrounding the concepts of screening procedures, bloodborne pathogens, donor selection, and gay, bisexual, trans, and other men who have sex with men populations (see Additional file 1). The search strategy borrowed concepts from a pre-existing strategy (11), but was modified to meet the specific objectives of this scoping review. The draft search strategy was peer-reviewed by a second information specialist using the PRESS checklist (12) before being finalized and translated across all databases. Searches were run on October 8, 2020, in Medline (via Ovid), Embase (via Elsevier), the Cumulative Index of Nursing and Allied Health Literature (via EBSCO), Sociological Abstracts (via ProQuest), and Web of Science Core Collection (SCI-EXPANDED, $\mathrm{SSCl}, \mathrm{AHCl}, \mathrm{CPCl}-\mathrm{S}, \mathrm{CPCl}-\mathrm{SSH}$, and $\mathrm{ESCl}$ ) for empirical studies and conference proceedings. No date limits were applied to the search, but the English language filter was applied within each database before search results were exported.

\section{Selection process}

The search results were imported into Covidence for review by the research team. After duplicates were removed, the remaining results were screened by title and abstract, then by full text review. Each record was reviewed independently by two members of the research team.

\section{Data charting process}

Data were extracted into a custom-designed spreadsheet. Data extraction for each article was performed by an initial extractor, then verified by another team member. The headings used to chart data were modelled on Arksey and O'Malley's framework for scoping reviews (13). For each article, the extractor charted the country being analysed, the country's blood donor policy for MSM at the time of the study, study aims, study population, sample size, phenomena of interest, methodology/methods, outcomes, key findings, and recommendations. An additional column was included to chart any equity issues addressed by the studies.

\section{Synthesis of results}

Articles were categorized based on a priori themes related to the study's objectives, as well as emergent themes identified collectively by the research team.

\section{Results}


A total of 3,974 records were imported to Covidence for screening, from which 1,154 duplicates were removed. During title/abstract screening, 2,510 records were excluded as irrelevant. Of the 310 articles that underwent full-text screening, 260 were ultimately excluded. One article (14) meeting all inclusion criteria was identified by the review's authors based on their personal knowledge of the literature, and was deemed relevant for inclusion in the review. A total of 51 articles were included in the scoping review. Figure 1 presents the results of the data collection process as a PRISMA flow diagram (15).

Articles were categorized by emergent themes pertaining to methodology, study population, and phenomena of interest. Table 1 shows a breakdown of the categories. Note that the categories are nonexclusive and occasionally overlapping, with some papers categorized by multiple themes.

\section{Table 1: Categorization of included studies}

\begin{tabular}{|lll|}
\hline Category & $\begin{array}{l}\text { Number of Papers } \\
(\mathbf{n})(\mathbf{n = 5 1})\end{array}$ & $\begin{array}{l}\mathbf{n} \text { as } \\
\text { Percentage }\end{array}$ \\
\hline Study uses a mathematical model & 13 & $25.4 \%$ \\
\hline Study is a surveillance study & 6 & $11.7 \%$ \\
\hline Study population includes gbMSM & 16 & $31.3 \%$ \\
\hline $\begin{array}{l}\text { Study population includes include transgender, non-binary, and } \\
\text { gender diverse people }\end{array}$ & 2 & $3.9 \%$ \\
\hline Study population is general donor population & 13 & $25.4 \%$ \\
\hline Study population is donor centre staff & 1 & $1.9 \%$ \\
\hline Study population is general population & 2 & $3.9 \%$ \\
\hline Study examines donor policies & 2 & $3.9 \%$ \\
\hline
\end{tabular}

\section{Mathematical models and surveillance studies}

Mathematical models and surveillance studies represent $38 \%$ of the studies included. Blood operators and regulators focus on mathematical models and surveillance studies to predict risk (mathematical models) associated with various changes to deferral period and policies and assess "real world" impact of any changes (surveillance studies). The fact that these studies make up the largest percentage of studies supports the view that blood operators and regulators prioritize safety considerations with any potential changes to donor screening and eligibility policies. 
As a category with high relevance to the study's objectives, articles whose study population includes gbMSM were sub-categorized by emergent themes (see Table 2). Note that some papers are included in multiple categories. Charted data pertaining to these sub-categories are included in Additional file 2.

\section{Table 2: Sub-categorization of studies whose study population included gbMSM}

\begin{tabular}{|l|l|}
\hline Category & $\begin{array}{l}\text { Number of } \\
\text { Papers }\end{array}$ \\
\hline Study population also includes non-gbMSM & 4 \\
\hline Study examines willingness to donate & 4 \\
\hline Study examines donation practices & 2 \\
\hline Study examines eligibility to donate & 1 \\
\hline $\begin{array}{l}\text { Study examines views/attitudes on different screening and eligibility criteria for } \\
\text { gbMSM }\end{array}$ & 6 \\
\hline
\end{tabular}

Table 3, Additional file 2 summarizes the data charted in studies (16-19) that included both gbMSM and non-gbMSM in the study population.

A further 12 papers focused exclusively on a gbMSM study population. Of these papers, 4 (20\%) focused on willingness to donate $(2,20,21)$. Table 4 , Additional file 2 summarizes these studies.

Table 5, Additional file 2 summarizes the studies $(22,23)$ that examined a gbMSM study population with regards to donation practices.

Table 6, Additional file 2 summarizes the paper (24) that examined a gbMSM study population with regards to eligibility to donate.

Table 7, Additional file 2 summarizes the papers $(3,25-28)$ that examined a gbMSM study population with regards to views and attitudes on different screening and eligibility criteria for gbMSM.

\section{Transgender, non-binary and gender diverse study population}

Only three papers included transgender, non-binary, and gender diverse study populations: Clackett et al, 2020 (27), Grace et al, 2020 (2), and Caruso et al, 2019 (14). Both studies were included in the tabular results above for gbMSM study populations. Clackett et al (2020) mention that transgender men were included in the study but does not provide any specific results related to transgender participants. In Grace et al, their qualitative investigation of willingness to donate among Canadian gbMSM revealed particular frustration expressed by transgender men $(n=3)$. Authors note that this differential response by 
transgender men suggests the need for further research among this population. Caruso et al (2019) include two people who identify as queer/genderfluid/nonbinary, and one trans man; however, results specific to these participants are not specified.

\section{Other study populations}

One study (29) examined blood centre staff perspectives on permanent deferral for gbMSM and potential challenges surrounding a change to 12-month deferral in the United States. Two papers, Go et al, 2011 (30) and Romeijn et al, 2018 (17), included the general (non-donor) population in the study. Two additional studies focused on donor policies: Schink et al, 2018 (31) examined gbMSM donor deferral policies across Europe and Cascio and Yomtovian, 2013 (32) conducted discourse analysis of the construction of gbMSM and HIV transmission in donor education materials.

\section{Discussion}

The scoping review revealed that most studies that examine gbMSM views are from the USA and take a quantitative, mathematical modelling approach. Many focus on determining what percentage of gbMSM have donated blood or plasma under various deferral time periods. This suggests that the prevailing research focus is on understanding the effectiveness of time-based deferral policies and potentially determining "compliance" of gbMSM with these policies. These studies mainly seemed to be assessing the effectiveness of criteria that screen out gbMSM donors versus looking for meaningful ways to include these donors in donation.

We observed a reliance on mathematical modeling and behavioural risk surveillance as a means of continuing with the status quo in donor policies which continue to ban gbMSM from donating. Without calling into question the mathematical basis of the models themselves, it is seldom clear from these studies how these numerical measures of risk translate into real-world decisions around risk mitigation. As Vamvakas notes (33), gbMSM and transgender donors are not the only source of risk to the blood supply, yet in practice this risk seems to be tolerated differently than others. It may be that blood operators are prioritizing a particular conception of safety to the exclusion of other important equityrelated considerations.

The absence of equity considerations from the studies in this scoping review is noteworthy. Though $30 \%$ of studies included gbMSM as participants, in general they do not consider equity issues or take an intersectional approach. Only 6 studies $(12 \%)$ mention any equity considerations. Notable among these are two qualitative studies by Grace et al, which include calls for more intersectional research (2) and discussion of the repair work that blood operators must do to address the cumulative effects of restrictive donor policies on gbMSM communities (3). 
It is noteworthy that relevant papers with an equity focus exist but did not meet the specific inclusion criteria for this scoping review, largely because they are embedded within social science rather than biomedical and scientific literature. It is imperative that equity considerations not remain siloed from other types of discussion around blood donation, but rather be incorporated into all types of research on blood donation policy as a matter of course.

Crucially, our scoping review confirmed a significant lack of research on transgender, non-binary, and gender-diverse people and blood donation. Only two studies (4\%) include transgender individuals in their methodology and only one study specifically described and quantified how transgender people were included in the study population. Few of the remaining studies make mention of transgender, non-binary, and gender diverse populations at all. Given the significant concerns of transgender donors with current screening practices, including being denied donation all together, being misgendered in screening, and being asked differential and invasive screening questions that are not asked of other donors, this lack of inclusion of transgender people and the intersectionality of how the "MSM deferral" criteria impacts transgender populations is concerning. Considering Canadian Blood Services' intention to make its policies and processes more inclusive of gender minorities, this gap constitutes an urgent area for further research.

There was also a dearth of research that specifically considered about plasma donation. Only two published studies specifically examined gbMSM views on plasma donation $(2,14)$. Given the direction that Canadian Blood Services is intending to take with the Fall 2021 Health Canada submission to allow sexually active gbMSM in long term monogamous relationships to donate blood and the worldwide shortage of plasma $(34,35)$ to manufacture into purified plasma protein products, this dearth of plasma related research is concerning.

Consistently, studies that examine the willingness of gbMSM to donate if policies change and/or they become eligible find that gbMSM indicate a high degree of willingness to donate. However, it is likely that years of exclusionary donor policies have created mistrust and resentment towards CBS within gbMSM, transgender, non-binary, gender diverse, and racially diverse communities. Reparations with these communities will be necessary to convert willingness to donate into real action once policies are changed. Subsequent phases of this research will aim to inform this goal through both qualitative and quantitative research on current attitudes within these communities about current donor policies and needed future changes to these policies.

\section{Limitations}

It is worth noting there are some limitations in this scoping review. The choice to screen papers in English only introduces a language bias that could impact the inclusion of relevant evidence, especially since this topic focuses on OECD countries. In addition to this, a grey literature search was not formally conducted to locate additional materials from key stakeholders and other relevant organizations. Future reviews could include such materials to compare the positions of relevant organizations with conclusions from 
published scholarly literature. Finally, stakeholder consultation was not included as part of this review, but subsequent phases of this research will seek to include those voices in the discussion on this topic.

\section{Conclusions}

In summary, it is noteworthy that many OECD countries are revising their blood donor policies affecting gbMSM based on current science related to risk. The shift away from behavioural surveillance targeting gbMSM is seen as an important step forward. However, how the broader uptake of such an approach among blood operators will occur is still not fully understood. Further, the overall lack of focus on the unique needs and equity considerations facing transgender populations requires further examination in order to ensure the next iteration of blood and plasma donor policies include their concerns.

\section{Declarations}

Ethics and consent to participate: Not applicable

Consent for publication: Not applicable

Availability of data and materials: All data generated or analysed during this study are included in this published article.

Competing interests: The authors declare that they have no competing interests.

Funding: This research received funding support from Canadian Blood Services MSM Plasma Program, funded by the federal government (Health Canada) and the provincial and territorial ministries of health. The views herein do not necessarily reflect the views of the federal, provincial, or territorial governments of Canada.

Authors' contributions: JG designed and led the research project, screened studies for inclusion, charted data, and drafted and edited the final manuscript. JH and TF contributed to research design, screened studies for inclusion, charted data, and drafted and edited the final manuscript. MR screened studies for inclusion, charted data, and drafted the final manuscript. CF drafted and edited the final manuscript. CJ coordinated the community advisory committee, provided stakeholder input, and contributed to research design. JP developed the search strategy, conducted searches of databases, and contributed to the final 
manuscript. Members of the Blood \& Plasma Donation Research Group contributed to research design and direction. All authors read and approved the final manuscript.

Acknowledgements: The authors wish to thank the additional members of the Blood \& Plasma Donation Research Group (OmiSoore Dryden, Eli Manning, Mike Mullowney, Sheila O'Brien, Todd Hatchette, Nathan Lachowsky, and Landon Getz) for their research contributions, as well as Leah Boulos, Senior Evidence Synthesis Consultant (Maritime SPOR SUPPORT Unit) for peer-reviewing the electronic search strategy. The authors also wish to thank the members of this project's community advisory committee for their valuable input.

\section{Abbreviations}

CBS $=$ Canadian Blood Services

gbMSM = gay, bisexual, and other men who have sex with men

$\mathrm{OECD}=$ Organization for Economic Co-operation and Development

\section{References}

1. Canadian Blood Services. Evolving eligibility criteria for gay, bisexual and other men [Internet]. Ottawa, ON: Canadian Blood Services. [cited 2021 Oct 18]. Available from:

https://www.blood.ca/en/blood/am-i-eligible-donate-blood/men-who-have-sex-men/eligibility-criteria-gaybisexual-and-other

2. Grace D, Gaspar M, Klassen B, Lessard D, Brennan DJ, Lachowsky NJ, et al. It's in me to give: Canadian gay, bisexual, and queer men's willingness to donate blood if eligible despite feelings of policy discrimination. Qual Health Res. 2020 Dec;30(14):2234-47.

3. Grace D, Gaspar M, Lessard D, Klassen B, Brennan DJ, Adam BD, et al. Gay and bisexual men's views on reforming blood donation policy in Canada: a qualitative study. BMC Public Health. 2019 Dec;19(1):772. doi:10.1177/1049732320952314.

4. Dryden 0. "A queer too far": Blackness, "gay blood", and transgressive possibilities. In: Walcott R, Dryden $\mathrm{O}$, Lenon S, editors. Disrupting queer inclusion: Canadian homonationalisms and the politics of belonging. Vancouver: UBC Press; 2015. p. 116-32.

5. Butler-Foster T, Chin-Yee I, Huang M, Jackson KT. Toward understanding culturally sensitive care for transgender blood donors: a scoping review of health care provider knowledge. Transgender Health. 2020 Jun 1;5(2):104-15. doi:10.1089/trgh.2019.0062. 
6. Goldman M, Butler-Foster T, Lapierre D, O'Brien SF, Devor A. Trans people and blood donation. Transfus. 2020;60(5):1084-92. doi:10.1111/trf.15777.

7. Pandey S, Gorlin JB, Townsend M, Van Buren N, Leung JNS, Lee C, et al International Forum on Gender Identification and Blood Collection: summary. Vox Sang. 2021 Sep 20;vox.13192. doi:10.1111/vox.13192.

8. Zeidler M. B.C. donor with "X" gender calls out Canadian Blood Services for refusing donation. CBC News [Internet]. 2019 Jul 27 [cited 2021 Nov 9]. Available from: https://www.cbc.ca/news/canada/britishcolumbia/x-gender-blood-donor-refused-1.5225682

9. Strapagiel L. Canadian Blood Services has a new policy that's being called anti-trans. BuzzFeed News [Internet]. 2016 Jun 21 [cited 2021 Nov 9]. Available from: https://www.buzzfeed.com/laurenstrapagiel/trans-canadians-blood-donation

10. Birrell S. Transphobia baked into policy at Canadian Blood Services. The Carillon [Internet]. 2021 Aug 5 [cited 2021 Nov 9]. Available from: https://www.carillonregina.com/transphobia-baked-into-policyat-canadian-blood-services/

11. McLean K. 2SLGBTQIA+ health: researchers [Internet]. Halifax, NS: Nova Scotia Health Authority Library Services. [cited 2020 Sept 23]. Available from: https://library.nshealth.ca/LGBTQ/Researchers

12. McGowan J, Sampson M, Salzwedel DM, Cogo E, Foerster V, Lefebvre C. PRESS Peer Review of Electronic Search Strategies: 2015 guideline statement. J Clin Epidemiol. 2016 Jul;75:40-6. doi:10.1016/j.jclinepi.2016.01.021.

13. Arksey H, O'Malley L. Scoping studies: towards a methodological framework. Int J Soc Res Methodol. 2005 Feb;8(1):19-32. doi:10.1080/1364557032000119616.

14. Caruso J, Germain M, Godin G, Myhal G, Pronovost F, Morin M, et al. 'One step closer': acceptability of a programme of plasma donation for fractionation from men who have sex with men. Vox Sang. 2019 Oct;114(7):675-86. doi:10.1111/vox.12827.

15. Page MJ, McKenzie JE, Bossuyt PM, Boutron I, Hoffmann TC, Mulrow CD, et al. The PRISMA 2020 statement: an updated guideline for reporting systematic reviews. BMJ. 2021 Mar 29;n71. doi:10.1136/bmj.n71.

16. Willson S, Miller K, Seem D, Kuehnert MJ. Cognitive evaluation of the AABB Uniform Donor History Questionnaire. Transfus. 2016 Jun;56(6pt2):1662-7. doi:10.1111/trf.13587.

17. Romeijn B, Merz E-M, Kok G, de Kort W, van Dongen A. Eligibility and willingness to donate blood in men who have (had) sex with men. Transfus. 2018 Mar;58(3):710-7. doi:10.1111/trf.14469. 
18. van Bilsen $\mathrm{WPH}$, Zaaijer HL, Matser A, van den Hurk K, Slot E, Schim van der Loeff MF, et al. Infection pressure in men who have sex with men and their suitability to donate blood. Clin Infect Dis. 2019 Mar 5;68(6):1001-8. doi:10.1093/cid/ciy596.

19. van de Laar TJ, Bezemer D, van Laethem K, Vandewalle G, de Smet A, van Wijngaerden E, et al. Phylogenetic evidence for underreporting of male-to-male sex among human immunodeficiency virusinfected donors in the Netherlands and Flanders. Transfus. 2017 May;57(5):1235-47. doi:10.1111/trf.14097.

20. Liszewski W, Becerril J, Terndrup C, West N, Lavin BC, Schieffler D, et al. The rates, perceptions, and willingness of men who have sex with men to donate blood. Transfus. 2014 Jul;54(7):1733-8. doi:10.1111/trf.12535.

21. Belanger GA, McFarland W, Raymond HF, Custer B. If the permanent deferral were lifted would men who have sex with men want to donate blood, and if so, who would be eligible? Transfus. 2013 Nov;53(11):2729-33. doi:10.1111/trf.12124.

22. Wentz AE, Merchant RC, Clark MA, Liu T, Rosenberger JG, Bauermeister JA, et al. Blood donation, sexual practices, and self-perceived risk for HIV in the United States among young adult men who have sex with men. Public Health Rep. 2019 Jan;134(1):36-46. doi:10.1177/0033354918815182.

23. Hughes S, Sheon N, Siedle-Khan B, Custer B. Saving lives, maintaining safety, and science-based policy: qualitative interview findings from the Blood Donation Rules Opinion Study (Blood DROPS). Transfus. 2015 Dec;55(12):2835-41. doi:10.1111/trf.13268.

24. Liszewski W, Terndrup C, Jackson NR, Helland S, Lavin BC. The beliefs and willingness of men who have sex with men to comply with a one-year blood donation deferral policy: a cross-sectional study. Transfus. 2017 Sep;57(9):2234-9. doi:10.1111/trf.14217.

25. Levy I, Olmer L, Livnat Y, Shalhavi R, Hizki O, Shinar E. Attitudes and perceptions among men having sex with men towards a new non-deferral blood donation policy in Israel. Vox Sang. 2019 May;114(4):310-6. doi:10.1111/vox.12766.

26. Levy I, Olmer L, Livnat Y, Yanko A, Shinar E. Attitudes, perceptions and knowledge among men who have sex with men towards the blood donation deferral policy in Israel. PLOS ONE. 2017 Feb 2;12(2):e0170364. doi:10.1371/journal.pone.0170364.

27. Clackett S, Seed CR, Prestage G, Hammoud MA, Hoad V, Saxton P, et al. Attitudes and willingness to donate blood among gay and bisexual men in Australia. Transfus. 2020 May;60(5):965-73. doi:10.1111/trf.15768.

28. Custer B, Murcia K, Robinson WT, McFarland W, Raymond HF. Blood donation history and eligibility assessment in a community-based sample of men who have sex with men. Transfus. 2018 
29. Hughes SD, Custer B, Laborde N, Sheon N. Transition to a 1-year deferral for male blood donors who report sexual contact with men: staff perspectives at one blood collection organization. Transfus. 2018 Aug;58(8):1909-15. doi:10.1111/trf.14632.

30. Go SL, Lam CTY, Lin YT, Wong DJ, Lazo-Langner A, Chin-Yee I. The attitude of Canadian university students toward a behavior-based blood donor health assessment questionnaire. Transfus. 2011 Apr;51(4):742-52. doi:10.1111/j.1537-2995.2010.02951.x.

31. Schink SB, Offergeld R, Schmidt AJ, Marcus U. Blood donor deferral policies across Europe and characteristics of men who have sex with men screened for human immunodeficiency virus in blood establishments: data from the European Men-who-have-sex-with-men Internet Survey (EMIS). Blood Transfus. 2018 Jan 1;16:7-16. doi:10.2450/2017.0109-16.

32. Cascio MA, Yomtovian R. Sex, risk, and education in donor educational materials: review and critique. Transfus Med Rev. 2013 Jan;27(1):50-5. doi:10.1016/j.tmrv.2012.05.003.

33. Vamvakas EC. Relative risk of reducing the lifetime blood donation deferral for men who have had sex with men versus currently tolerated transfusion risks. Transfus Med Rev. 2011 Jan;25(1):47-60. doi:10.1016/j.tmrv.2010.08.006.

34. Vesnaver E, Goldman M, O'Brien S, MacPherson P, Butler-Foster T, Lapierre D, et al. Barriers and enablers to source plasma donation by gay, bisexual and other men who have sex with men under revised eligibility criteria: protocol for a multiple stakeholder feasibility study. Health Res Policy Syst. 2020 Dec;18(1):131. doi:10.1186/s12961-020-00643-4.

35. Grace D, Gaspar M, Klassen B, Lessard D, Anand P, Brennan DJ, et al. Stepping stones or second class donors?: a qualitative analysis of gay, bisexual, and queer men's perspectives on plasma donation policy in Canada. BMC Public Health. 2021 Dec;21(1):444. doi:10.1186/s12889-021-10480-x.

\section{Tables}

Tables 3 - 7 are available in the supplementary files.

\section{Figures}




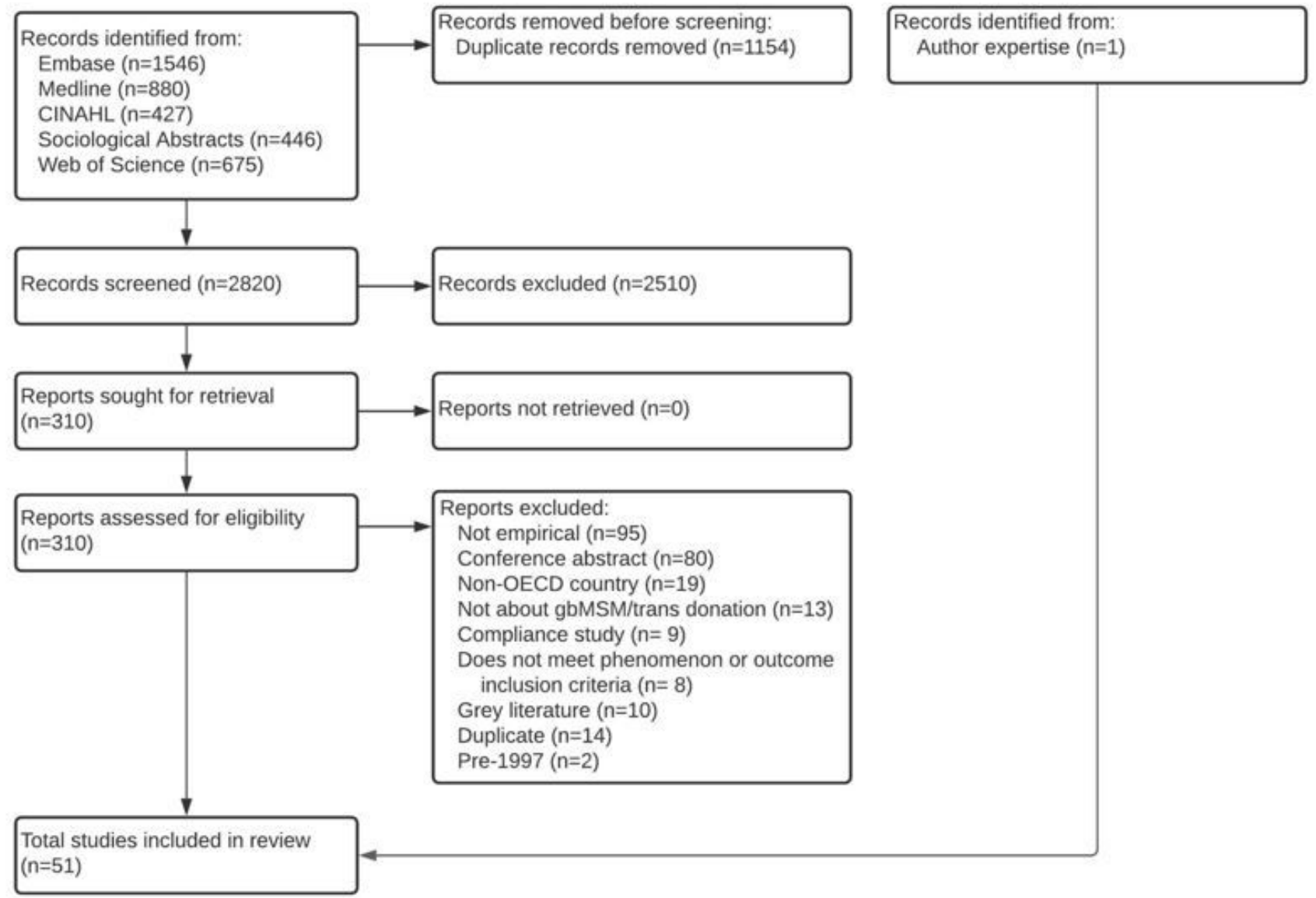

Figure 1

PRISMA flow diagram of search and screening process

\section{Supplementary Files}

This is a list of supplementary files associated with this preprint. Click to download.

- Additionalfile1.docx

- Additionalfile2.docx 